\title{
Central Haemodynamic Variability During Sleep in Subjects with and without Atrial Fibrillation
}

\author{
Michał Sitarek ${ }^{1}$, Gerard Cybulski ${ }^{1,2}$, Anna Gąsiorowska ${ }^{2}$, Ewa Ziółkowska ${ }^{2}$, Wiktor Niewiadomski ${ }^{2}$, \\ Anna Strasz ${ }^{2}$ \\ ${ }^{1}$ Institute of Metrology and Biomedical Engineering, Department of Mechatronics, \\ Warsaw University of Technology, Warsaw, Poland \\ ${ }^{2}$ Department of Applied Physiology, Mossakowski Medical Research Centre, \\ Polish Academy of Sciences, Warsaw, Poland
}

\begin{abstract}
The aim of this study was to develop a Matlab programme for quantitative evaluation and graphical presentation of haemodynamic variability parameters calculated from electrocardiography (ECG) and impedance cardiography (ICG) signals. We also sought to identify and compare patterns of variability in healthy subjects $(N)$ and subjects with atrial fibrillation $(A F)$.

We analysed 3000 seconds of ECG and ICG signals acquired continuously during overnight, home recordings of six healthy male subjects (20-22, group N) and five male patients with atrial fibrillation (ages 56-78, group $A F)$. Haemodynamic variability was determined using standard deviation and indices describing asymmetry of stroke volume, systolic time intervals (STI), and their derivatives. Stroke volume (SV), amplitude of impedance signal (dz/dtmax), ejection time (ET) and pre-ejection period $(P E P)$ were calculated automatically.

The standard deviation (SD) of $S V$ varied in the range of 9-16 $\mathrm{ml}$ in normal subjects, versus 12-39 $\mathrm{ml}$ in AF subjects. SD for ET was 14-42 $\mathrm{ms}$ for $N$ and 31-68 $\mathrm{ms}$ for $A F$. PEP SD varied 8-33 $\mathrm{ms}$ for $N$ and $12-38 \mathrm{~ms}$ for $A F$.

Preliminary results show that the patterns of haemodynamic variability in AF patients differed from those observed in non-patients for most of the analysed indices.
\end{abstract}

\section{Introduction}

\subsection{Motivation}

The analysis of variability of cardiovascular parameters might yield diagnostic data on autonomic control [1,2]. Cardiac rhythm variability has been intensively studied in various physiological and pathological situations, but only limited data are available regarding fluctuations of haemodynamic parameters, especially during overnight recordings.

Cybulski et al. [3] analysed central haemodynamic variability in a small group of subjects who either had atrial fibrillation or were healthy. It was concluded that the variability of SV, estimated by the coefficient of variation, was mainly caused by changes in the amplitude of the signal and that variation in ET had a smaller effect.

Siebert et al. [4] analysed stroke volume variability and heart rate power spectrum in relation to posture changes in healthy subjects. They concluded that the combined analysis of heart rate variability (HRV) and stroke volume variability (SVV) revealed different cardiovascular responses to postural stress in the three age groups considered.

Beat-to-beat variability of stroke volume output velocity (SVOV) at rest has also been measured in healthy subjects and in patients 10-14 days after acute myocardial infarction [5].

Holme et al. [6] proposed to use SV and SV variability SVV as clinical tools for detection of acute haemorrhage. They concluded that variability in stroke volume was decreased during mild and moderate hypovolemia and could be used for early detection of hypovolemia.

\subsection{Aim of the study}

The aim of this study was to develop a Matlab programme for quantitative evaluation and graphical presentation of haemodynamic variability parameters calculated from electrocardiography (ECG) and impedance cardiography (ICG) signals. We also sought to identify and compare patterns of variability in healthy subjects (N) and subjects with atrial fibrillation (AF). 


\section{Material and methods}

To estimate haemodynamic variability during sleep, we used data from our impedance cardiography signal database. These data were originally collected for other studies in order to analyse cardiac haemodynamics in atrial fibrillation patients [3] and in young healthy subjects participating in bedrest studies which were not included in the final paper [7].

\subsection{Subjects and procedure}

We analysed ECG and ICG signals acquired continuously during overnight, home recordings of six healthy male subjects (20-22 yrs., group N) and five male patients with atrial fibrillation (56-78 yrs., group AF). Haemodynamic variability was determined using standard deviation and indices describing asymmetry of stroke volume, systolic time intervals (STI) and their derivatives. For analysis, we used 3000 seconds of sleeptime recordings. Haemodynamic parameters - stroke volume (SV), amplitude of impedance signal (dz/dtmax), ejection time (ET), pre-ejection period (PEP) - were calculated off-line using our computer programme for automatic determination of SV using the Kubicek formula [8]. We visualised the variability of each parameter with a Poincare plot and calculated descriptors of short-term and long-term variability (SD1 and SD2, respectively).

\subsection{Methods and instrumentation}

We used a (previously described) wearable, ambulatory impedance cardiography recorder (Reomonitor) $[9,10]$. This device was constructed for non-invasive acquisition of central haemodynamic data during everyday activity. The analogue part of the Reomonitor consists of a one-channel ECG and a miniaturized impedance cardiograph. Changes in the thoracic impedance, reflecting SV, were evaluated using the tetrapolar method. An alternating current (with an effective amplitude of $1 \mathrm{~mA}$ and a frequency of $100 \mathrm{kHz}$ ) oscillated between the application electrodes while the voltage (reflecting the impedance) was measured between the receiving electrodes. ECG and the first derivative of the impedance cardiography signal (dz/dt) were sampled at $200 \mathrm{~Hz}$ with 8-bit resolution. Stroke volume was evaluated using the Kubicek formula [8]. The validity and reliability of impedance cardiography have been reviewed many times $[9,10]$. Ambulatory monitoring with the Reomonitor was verified using echocardiography in both the supine and tilted positions [10].

\subsection{Data analysis}

Haemodynamic variability was evaluated using SD, coefficient of variation (CV), quartiles (Q1, Q3), Poincare plot descriptors (SD1, SD2) and Guzik's index [11]. Guzik's index is a measure of asymmetry define by formula (1),

$$
G I=\frac{\sum_{i=1}^{C\left(P_{i}^{+}\right)}\left(D_{i}^{+}\right)^{2}}{\sum_{i=1}^{N-1}\left(D_{i}\right)^{2}} \times 100 \%
$$

This is the ratio of the sum of squared distances between a point and the line of identity for points lying above the line of identity on a Poincare plot to the sum of squares of distances of all points.

Artefacts were removed from the analysis based on the clearly defined criteria for each variable, determined mainly using physiological and pathophysiological ranges.

\section{Results}

In Tables 1-4 haemodynamic variables and their variability descriptors are presented for RR intervals, SV, cardiac output (CO) and dz/dt of ICG signal, respectively. Coefficient of variation are presented in percents, while Guzik's index is dimensionless. Other descriptors are presented in units of the measured variable. An asterisk indicates that the difference between groups $\mathrm{AF}$ and $\mathrm{N}$ for a given parameter is statistically significant at the level of $\mathrm{p}<0.05$.

Table 1. Parameters describing RR intervals [ms] and their variability for healthy subjects $(\mathrm{N})$ and patients with atrial fibrillation $(\mathrm{AF})$.

\begin{tabular}{lll}
\hline RR descriptor & $\mathrm{N}$ & $\mathrm{AF}$ \\
\hline Mean & 1116 & 915 \\
Median & 1108 & 701 \\
First quartile Q1 & 1042 & 855 \\
Third quartile Q3 & 1165 & 979 \\
Standard deviation & 97 & 325 \\
Coefficient of variation & 8.7 & 35.5 \\
SD1 (short term) & 51 & 90 \\
SD2 (long term) & 102 & 115 \\
Guzik's index & 46.4 & $49.5^{*}$ \\
\hline
\end{tabular}

Table 2. Parameters describing stroke volume $[\mathrm{ml}]$ and their variability for healthy subjects $(\mathrm{N})$ and patients with atrial fibrillation $(\mathrm{AF})$.

\begin{tabular}{lll}
\hline SV descriptor & N & AF \\
\hline Mean & 53 & 64 \\
Median & 52 & 62 \\
First quartile Q1 & 44 & 52 \\
Third quartile Q3 & 61 & 73 \\
Standard deviation & 12 & 17 \\
Coefficient of variation & 23 & 26 \\
SD1 (short term) & 10 & $14^{*}$ \\
SD2 (long term) & 13 & 17 \\
\hline
\end{tabular}


Guzik's index

49.1

49.4

Table 3. Parameters describing cardiac output [1/min] and their variability for healthy subjects $(\mathrm{N})$ and patients with atrial fibrillation (AF).

\begin{tabular}{lll}
\hline CO descriptor & $\mathrm{N}$ & $\mathrm{AF}$ \\
\hline Mean & 2.93 & 3.98 \\
Median & 2.83 & 3.86 \\
First quartile Q1 & 2.42 & 3.30 \\
Third quartile Q3 & 3.27 & 4.54 \\
Standard deviation & 0.69 & 0.96 \\
Coefficient of variation & 23.6 & 24.1 \\
SD1 (short term) & 0.56 & $0.98^{*}$ \\
SD2 (long term) & 0.79 & $1.21^{*}$ \\
Guzik's index & 48.5 & 49.4 \\
\hline
\end{tabular}

Table 4. Parameters describing first derivative amplitude $(\mathrm{dz} / \mathrm{dt})[\Omega / \mathrm{s}]$ and their variability for healthy subjects $(\mathrm{N})$ and patients with atrial fibrillation (AF).

\begin{tabular}{lll}
\hline dz/dt descriptor & $\mathrm{N}$ & $\mathrm{AF}$ \\
\hline Mean & 1.78 & $1.04^{*}$ \\
Median & 1.76 & 0.95 \\
First quartile Q1 & 1.62 & 0.85 \\
Third quartile Q3 & 1.95 & 1.13 \\
Standard deviation & 0.27 & 0.29 \\
Coefficient of variation & 15.2 & 27.9 \\
SD1 (short term) & 0.23 & 0.25 \\
SD2 (long term) & 0.30 & 0.31 \\
Guzik's index & 48.9 & 50.2 \\
\hline
\end{tabular}

Figure 1 presents a Poincare plot for SV variability in N3, a healthy subject. Figure 2 presents a Poincare plot for $\mathrm{SV}$ variability in $\mathrm{AF} 4$, an atrial fibrillation patient.

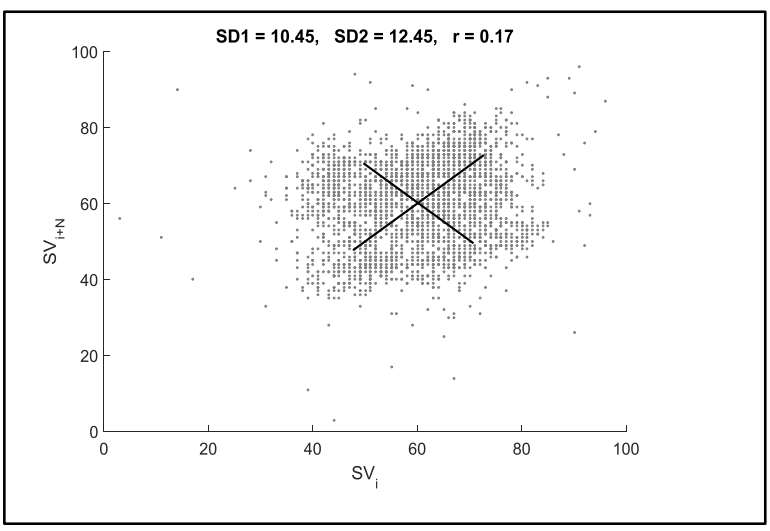

Figure 1. Poincare plot for stroke volume (SV) variability in a healthy subject (N3). SD1 and SD2 represent the Poincare plot descriptors.

The short-term variability index for SV (SD1) was significantly higher in patients with atrial fibrillation than in healthy subjects. For CO, both SD1 and SD2, were higher in patients with atrial fibrillation.

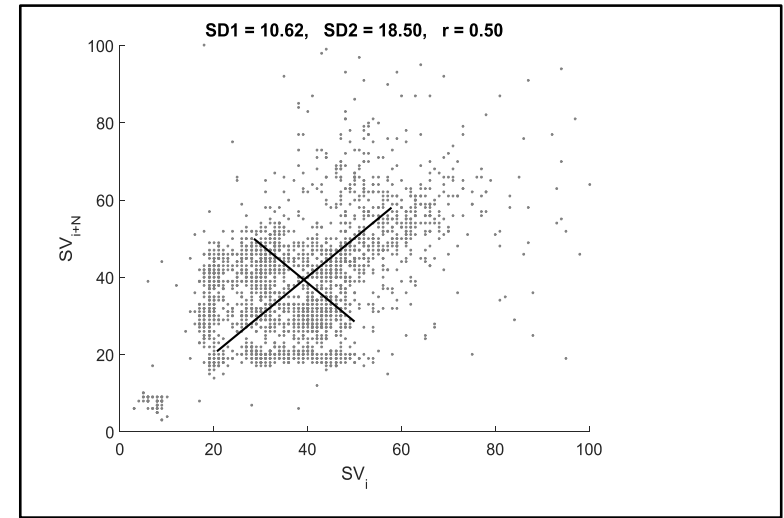

Figure 2. Poincare plot for stroke volume (SV) variability in an atrial fibrillation patient (AF4). SD1 and SD2 represent the Poincare plot descriptors.

The Guzik's indices did not differ between the two groups for most haemodynamic parameters, except that for RR intervals, for which the index for $A F$ was significantly higher.

\section{Discussion and conclusions}

The advantages/disadvantages and application perspectives of the ICG method have been presented in several reviews $[12,13,14]$. Fellahi and Fisher [15] noted that, despite some disappointing results for the measurement of absolute values of cardiac output relative to some reference methods, ICG might be "interesting in guiding the spontaneous or induced changes in cardiac output and medical decision-making at the bedside". Cybulski [12] pointed out the usefulness of ambulatory ICG in the non-invasive assessment of haemodynamic impairment caused by cardiac arrhythmias, verification of VVI pacemakers and optimisation of AV delay in dualchamber pacing systems during normal daily activity. It was also emphasised that an ambulatory version of ICG could be used to record transient events, which would be difficult or even impossible to visualize using other, established, "classical" methods. Some perspectives of ICG development were presented in Cybulski et al. $[12,13]$.

The main research limitation of the study is the small size of the sample. Another is associated with uncertainty of the method, regarding the limitations of the proper detection of the B-point on the dz/dt curve in ICG [15]. Possible uncertainty in detecting the X-point on the ICG curve was also recently reported [16]. This results in uncertainty in determination of ejection time (ET), which directly affects SV calculation. We feel unable to estimate 
the impact of variation in systolic time intervals caused by method uncertainties in the variation induced by physiological changes. We could only assume that Bdetection and X-detection uncertainty are constant for a given subject.

We developed a tool for quantitative and graphical presentation of haemodynamic variability. For a small sample, preliminary results show the patterns of haemodynamic variability in AF patients differing from those observed in non-patients for most of the analysed indices.

\section{Acknowledgements}

The study was supported by the research programs of institutions the authors are affiliated with. We also thank all people helping us to acquire data for principal studies $[3,7]$.

\section{References}

[1] Cybulski G. Influence of age on the immediate cardiovascular response to the orthostatic maneouvre. European Journal of Applied Physiology 1996;73:563-72.

[2] Koźluk E, Cybulski G, Piątkowska A, Zastawna I, Niewiadomski W, Strasz A, Gąsiorowska A, Kempa M, Kozłowski D, Opolski G. Early hemodynamic response to the tilt test in patients with syncope. Arch Med Sci 2014;10(6):1078-85.

[3] Cybulski G, Ziółkowska E, Książkiewicz A, Łukasik W, Niewiadomski W, Kodrzycka A, Pałko T. Application of Impedance Cardiography Ambulatory Monitoring Device for Analysis of Central Hemodynamics Variability in Atrial Fibrillation. Computers in Cardiology 1999;26:563-6.

[4] Siebert J, Drabik P, Lango R, Szyndler K. Stroke volume variability and heart rate power spectrum in relation to posture changes in healthy subjects. Med Sci Monitor 2004; 10(2):MT31-7.

[5] Zubarev MA, Schekotov VV, Parandey OR, Dumler AA. Beat - to - beat variability of stroke volume output velocity measured by an impedance cardiographic method. IFMBE Proceedings 2007; 17:579-81.

[6] Holme NLA, Rein EB, Elstad M. Cardiac stroke volume variability measured non-invasively by three methods for detection of central hypovolemia in healthy humans. Eur J Appl Physiol 2016;116(11-12):2187-96.

[7] Gąsiorowska A, Nazar K, Mikulski T, Cybulski G, Niewiadomski W, Smorawinski J, Krzeminski K, Porta S, Kaciuba-Uscilko H. Hemodynamic and neuroendocrine predictors of lower body negative pressure (LBNP) intolerance in healthy young men. J Physiol Pharmacol 2005, 56 (2): 179-193.
[8] Kubicek WG, Karnegis JN, Patterson RP, Witsoe DA, Mattson RH. Development and evaluation of an impedance cardiac output system. Aerospace Medicine 1966;37:120812.

[9] Cybulski G, Książkiewicz A, Łukasik W, Niewiadomski W, Pałko T. Ambulatory monitoring device for central hemodynamics and ECG signals recording on PCMCIA flash memory cards, Computers in Cardiology 1995;22:505-7.

[10] Cybulski G, Michalak E, Koźluk E, Piątkowska A, Niewiadomski W. Stroke volume and systolic time intervals: beat-to-beat comparison between echocardiography and ambulatory impedance cardiography in supine and tilted positions. Medical and Biological Engineering and Computing 2004;42:707-11.

[11] Guzik P., Piskorski J., Krauze T., Wykrętowicz A., Wysocki H., Heart rate asymmetry by Poincaré plots of RR intervals, Biomed Tech 2006, 51:272-275.

[12] Cybulski G. Ambulatory impedance cardiography: new possibilities (Letter to the Editor). J Appl Physiol 2000;88:1509-10.

[13] Cybulski G, Strasz A, Niewiadomski W, Gąsiorowska A. Impedance cardiography: Recent advancements. Cardiol J 2012;19(5):550-6.

[14] Fellahi JL, Fischer MO. Electrical bioimpedance cardiography: an old technology with new hopes for the future. J Cardiothorac Vasc Anesth 2014;28(3):755-60.

[15] Lozano DL, Norman G, Knox D, Wood BL, Miller BD, Emery CF, Berntson GG. Where to $B$ in $\mathrm{dZ} / \mathrm{dt}$. Psychophysiology 2007;44(1):113-19.

[16] Cybulski G, Młyńczak G, Żyliński M, Strasz A, Gąsiorowska A, Niewiadomski W. The Quality of Automatic Artifact Identification in Ambulatory Impedance Cardiography Monitoring. In: Eskola H., Väisänen O., Viik J., Hyttinen J. (eds) EMBEC \& NBC 2017. EMBEC 2017, NBC 2017. Singapore: Springer, IFMBE Proceedings, vol 65: $165-8$.

Address for correspondence.

Gerard Cybulski

Institute of Metrology and Biomedical Engineering

Department of Mechatronics

Warsaw University of Technology

Św. Andrzeja Boboli 8

02-525 Warsaw, Poland

G.Cybulski@mchtr.pw.edu.pl 